\title{
One loop partition function of three-dimensional flat gravity
}

\section{G. Barnich, ${ }^{a}$ H.A. González, ${ }^{a}$ A. Maloney ${ }^{b}$ and B. Oblak ${ }^{a}$}

${ }^{a}$ Physique Théorique et Mathématique, Université Libre de Bruxelles and International Solvay Institutes, Campus Plaine C.P. 231, B-1050 Bruxelles, Belgium

${ }^{b}$ McGill Physics Department, 3600 rue University, Montréal, QC H3A 2T8, Canada

E-mail: gbarnich@ulb.ac.be, hdgonzal@uc.cl, maloney@physics.mcgill.ca, boblak@ulb.ac.be

ABSTRACT: In this note we point out that the one-loop partition function of threedimensional flat gravity, computed along the lines originally developed for the anti-de Sitter case, reproduces characters of the $\mathrm{BMS}_{3}$ group.

Keywords: Conformal and W Symmetry, Space-Time Symmetries, Classical Theories of Gravity, Topological Field Theories

ARXIV EPRINT: 1502.06185 


\section{Contents}

1 Introduction 1

2 The set-up 1

3 Evaluation of the determinants 3

\section{Introduction}

Three-dimensional gravity has emerged as an important testing ground for our ideas about quantum gravity. Following earlier work $[1,2]$, it was shown that at one loop the partition function of $\mathrm{AdS}_{3}$ gravity is a character of the Virasoro algebra [3] (see [4] for an improved derivation and generalizations). This is in line with the analysis of the asymptotic symmetry group of $\mathrm{AdS}_{3}$ gravity by Brown and Henneaux [5], and is a direct check of the fact that, at the quantum mechanical level, the bulk gravity states organize into representations of the conformal group. In this note we point out that a similar one-loop computation in the technically simpler case of flat gravity reproduces the vacuum character of the $\mathrm{BMS}_{3}$ group. The latter is the symmetry group of asymptotically flat gravity at null infinity [6, 7], whose characters have been recently worked out in [8] (see also $[9,10]$ for details on $\mathrm{BMS}_{3}$ representations).

\section{The set-up}

We wish to study the gravitational partition function

$$
Z[\beta, \theta]=\int \mathcal{D} g e^{-\frac{1}{\hbar} S_{E}}, \quad S_{E}=-\frac{1}{16 \pi G} \int d^{3} x \sqrt{g} R+B
$$

Here $S_{E}$ is the Euclidean Einstein action (with no cosmological constant), $B$ is an appropriate boundary term and $\beta, \theta$ are the inverse temperature and angular potential. The parameters $\beta, \theta$ determine a quotient $\mathbb{R}^{3} / \mathbb{Z}$ of flat Euclidean space, representing "thermal spinning $\mathbb{R}^{3 "}$ :

$$
\begin{aligned}
d s^{2}=d x_{1}^{2}+d x_{2}^{2}+d x_{3}^{2} & =d y^{2}+d \rho^{2}+\rho^{2} d \varphi^{2}, \\
(y, \varphi) \sim \gamma(y, \varphi) & =(y+\beta, \varphi+\theta)
\end{aligned}
$$

where $\mathbb{Z}$ is the discrete group generated by the identification $\gamma$.

We wish to compute $Z[\beta, \theta]$ in a perturbative expansion around the background (2.2). The partition function (2.1) can be expanded as

$$
\hbar \ln Z[\beta, \theta]=-S^{(0)}+\hbar S^{(1)}+\hbar^{2} S^{(2)}+\ldots
$$


where $S^{(0)}$ is the classical action and $S^{(i)}$ an $i$-loop correction. We will focus on $S^{(0)}$ and $S^{(1)}$.

There are several ways to compute $S^{(0)}$ from the classical action, including boundary terms. The most direct is to use the Hamiltonian form of the action. In this case, the surface term at infinity is, by definition, determined by the surface charges times their chemical potentials $[12,13]$. For flat space, this is the only contribution and the on-shell action is automatically finite. Note however that the overall normalization of the charges is not fixed. In other words, requiring a well defined variational principe and a finite on-shell action for a class of spacetimes does not fix the ambiguity that consists in adding a finite combination of the quantities that are held fixed in the variational principle. An additional criterion is needed to lift this ambiguity.

In order to make the classical part of the partition function invariant under the analog of modular $S$-transformations in the flat case [14, 15]

$$
\beta \rightarrow \frac{4 \pi^{2} \beta}{\theta^{2}}, \quad \theta \rightarrow-\frac{4 \pi^{2}}{\theta},
$$

the charges have to be normalised with respect to the null orbifold [16], $d s^{2}=-2 d u d r+$ $r^{2} d \phi^{2}$. This puts mass and angular momentum of flat space at $M=-\frac{1}{8 G}, J=0[7]$, and the tree-level Euclidean action for flat space is

$$
S^{(0)}=-\frac{\beta}{8 G},
$$

a result originally derived along slightly different lines in [17]. For cosmological solutions [19-22] with metric

$$
d s^{2}=8 G M d u^{2}-2 d u d r+8 G J d u d \phi+r^{2} d \phi^{2}
$$

and $M \geqslant 0$, the Euclidean action takes the value

$$
S^{(0)}=-\frac{\pi^{2} \beta}{2 G \theta^{2}},
$$

which can be obtained from the flat space result (2.5) through the transformation (2.4).

One can then use the fact that three-dimensional gravity has no local degrees of freedom to understand the nature of the quantum corrections $S^{(i)}$ to this classical action, following [2]. One might naively think that - as there are no local degrees of freedom to run in loops - all loop corrections must vanish. This is not quite the case, however, since there may not be complete cancellation between the graviton loops and the ghost loops which arise from gauge fixing. In theories with topological degrees of freedom, such as ChernSimons theory, this is typically the case. Given the similarities between three-dimensional gravity and Chern-Simons theory we therefore expect that the $S^{(i)}$ may be non-trivial.

The perturbative contributions $S^{(i)}$ can in principle be obtained by quantizing a classical phase space, which in the present case is the space of metrics which are smoothly connected to the classical solution (2.2), modulo an appropriate group of diffeomorphisms. We must impose boundary conditions on the metric at asymptotic infinity, following [6, 7], 
which require that we only include metrics with finite values of the BMS charges. Only those diffeomorphisms which vanish sufficiently quickly at asymptotic infinity - in the sense that they do not change the values of these charges - are regarded as gauge transformations. One can then obtain the classical phase space by applying to the metric (2.2) the diffeomorphisms which do not vanish sufficiently quickly at infinity. Since there are no local degrees of freedom, the entire phase space of solutions which are continuously connected to (2.2) is constructed by this group of non-trivial diffeomorphisms. More precisely, since the transformation law of asymptotically flat metrics under $\mathrm{BMS}_{3}$ is given by the coadjoint representation, the perturbative contributions $S^{(i)}$ are obtained by quantizing the coadjoint orbit of flat space under the asymptotic symmetry group. The resulting Hilbert space will naturally be a representation of $\mathrm{BMS}_{3}$, and the partition function a character of $\mathrm{BMS}_{3}$.

We therefore expect that the perturbative partition function should be given by the vacuum $\mathrm{BMS}_{3}$ character described in [8]. This result is one loop exact: $S^{(1)} \neq 0$, but $S^{(i)}=0$ for all $i \geq 2$. The one-loop exactness of the perturbative gravity partition function was already observed in $\mathrm{AdS}_{3}$ gravity [2].

The above argument, while appealing, has the structure of the BMS group built in from the beginning. It would be preferable to verify this expectation from a direct gravitational computation, as this would give a check of the BMS structure of flat space gravity at the quantum level. We will now describe the direct computation of $S^{(1)}$, leaving higher loop corrections for future work.

\section{Evaluation of the determinants}

We follow closely [3], to which we refer for more details. The one-loop contribution to the partition function is given by

$$
S^{(1)}=-\frac{1}{2} \ln \operatorname{det} \Delta^{(2)}+\ln \operatorname{det} \Delta^{(1)}-\frac{1}{2} \ln \operatorname{det} \Delta^{(0)} .
$$

Here $\Delta^{(2)}, \Delta^{(1)}, \Delta^{(0)}$ are the kinetic operators which arise in the linearized expansion of general relativity around the flat background metric (2.2), including ghosts. They are the Laplacian operators for a massless, traceless symmetric tensor, a vector, and a scalar, respectively (see also e.g. [11]).

The determinants are evaluated using the heat kernel approach, which is straightforward in flat space. The heat kernels $K, K_{\mu \mu^{\prime}}$ and $K_{\mu \nu, \mu^{\prime} \nu^{\prime}}$ are defined to be solutions to the differential equations

$$
\begin{aligned}
\left(\Delta^{(0)}-m^{2}-\partial_{t}\right) K\left(t ; x, x^{\prime}\right) & =0, \\
\left(\Delta^{(1)}-m^{2}-\partial_{t}\right) K_{\mu \mu^{\prime}}\left(t ; x, x^{\prime}\right) & =0, \\
\left(\Delta^{(2)}-m^{2}-\partial_{t}\right) K_{\mu \nu, \mu^{\prime} \nu^{\prime}}\left(t ; x, x^{\prime}\right) & =0,
\end{aligned}
$$


with boundary conditions at $t=0$ :

$$
\begin{aligned}
K\left(0 ; x, x^{\prime}\right) & =\delta^{(3)}\left(x-x^{\prime}\right) \\
K_{\mu \nu^{\prime}}\left(0 ; x, x^{\prime}\right) & =\delta^{(3)}\left(x-x^{\prime}\right) \delta_{\mu \mu^{\prime}}, \\
K_{\mu \nu, \mu^{\prime} \nu^{\prime}}\left(0 ; x, x^{\prime}\right) & =\frac{1}{2}\left(\delta_{\mu \mu^{\prime}} \delta_{\nu \nu^{\prime}}+\delta_{\mu \nu^{\prime}} \delta_{\nu \mu^{\prime}}-\frac{2}{3} \delta_{\mu \nu} \delta_{\mu^{\prime} \nu^{\prime}}\right) \delta^{(3)}\left(x-x^{\prime}\right) .
\end{aligned}
$$

We have introduced a mass parameter $m$, so that these heat kernels encode the spectra of the massive kinetic operators $\Delta^{(i)}-m^{2}$. Defining the world function

$$
\sigma=\frac{1}{2}\left|x-x^{\prime}\right|^{2}
$$

the heat kernels are

$$
\begin{aligned}
K\left(t ; x, x^{\prime}\right) & =\frac{1}{(4 \pi t)^{\frac{3}{2}}} e^{-m^{2} t-\frac{\sigma}{2 t}} \\
K_{\mu \mu^{\prime}}\left(t ; x, x^{\prime}\right) & =-K\left(t ; x, x^{\prime}\right) \partial_{\mu} \partial_{\mu^{\prime}} \sigma \\
K_{\mu \nu, \mu^{\prime} \nu^{\prime}}\left(t ; x, x^{\prime}\right) & =\frac{1}{2} K\left(t ; x, x^{\prime}\right)\left(\partial_{\mu} \partial_{\mu^{\prime}} \sigma \partial_{\nu} \partial_{\nu^{\prime}} \sigma+\partial_{\mu} \partial_{\nu^{\prime}} \sigma \partial_{\nu} \partial_{\mu^{\prime}} \sigma-\frac{2}{3} \delta_{\mu \nu} \delta_{\mu^{\prime} \nu^{\prime}}\right) .
\end{aligned}
$$

The essential point is that the heat kernels obey linear differential equations, so given the heat kernels in flat space one can readily obtain the heat kernel in a quotient of flat space using the method of images. For thermal spinning $\mathbb{R}^{3}$ we have

$$
K^{\mathbb{R}^{3} / \mathbb{Z}}(t, x, x)=\sum_{n \in \mathbb{Z}} K\left(t, \sigma\left(x, \gamma^{n} x\right)\right)
$$

The Euclidean distance between a point and its $n$-th image under $\gamma$ is

$$
\sigma\left(x, \gamma^{n} x\right)=\frac{1}{2} n^{2} \beta^{2}+2 \sin ^{2}\left(\frac{n \theta}{2}\right) \rho^{2}
$$

so that

$$
K^{\mathbb{R}^{3} / \mathbb{Z}}(t, x, x)=K(t ; x, x)+\frac{2 e^{-m^{2} t}}{(4 \pi t)^{3 / 2}} \sum_{n=1}^{\infty} e^{-\sin ^{2}\left(\frac{n \theta}{2}\right) \frac{\rho^{2}}{t}-\frac{n^{2} \beta^{2}}{4 t}} .
$$

We can now extract the determinants of the operators $\Delta^{(i)}-m^{2}$ by integrating these heat kernels:

$$
\begin{aligned}
-\ln \operatorname{det}\left(\Delta^{(0)}-m^{2}\right) & =\int_{0}^{\infty} \frac{d t}{t} \int_{0}^{\beta} d y \int_{0}^{2 \pi} d \varphi \int_{0}^{\infty} \rho d \rho K^{\mathbb{R}^{3} / \mathbb{Z}}(t, x, x) \\
& =\frac{m^{3}}{6 \pi} \operatorname{Vol}\left(\mathbb{R}^{3} / \mathbb{Z}\right)+2 \sum_{n=1}^{\infty} \int_{0}^{\infty} d t(2 \pi \beta) \frac{e^{-m^{2} t}}{(4 \pi t)^{3 / 2}} \frac{e^{-\frac{n^{2} \beta^{2}}{4 t}}}{2 \sin ^{2}\left(\frac{n \theta}{2}\right)}, \\
& =\frac{m^{3}}{6 \pi} \operatorname{Vol}\left(\mathbb{R}^{3} / \mathbb{Z}\right)+\sum_{n=1}^{\infty} \frac{2 e^{-n \beta m}}{n\left|1-e^{i n \theta}\right|^{2}}
\end{aligned}
$$


Defining $\widehat{g}^{\mu \nu^{\prime}}=g^{\mu \rho} \partial_{\rho}\left(\gamma^{n} x\right)^{\nu^{\prime}}$ and using $\widehat{g}^{\mu \nu^{\prime}} \partial_{\mu} \partial_{\nu^{\prime}} \sigma=-1-2 \cos (n \theta)$,

$$
\begin{aligned}
-\ln \operatorname{det}\left(\Delta^{(1)}-m^{2}\right) & =\int_{0}^{\infty} \frac{d t}{t} \sum_{n \in \mathbb{Z}} \int d^{3} x \sqrt{g} \widehat{g}^{\mu \nu^{\prime}} K_{\mu \nu^{\prime}}\left(t, \sigma\left(x, \gamma^{n} x\right)\right) \\
& =\frac{m^{3}}{2 \pi} \operatorname{Vol}\left(\mathbb{R}^{3} / \mathbb{Z}\right)+\sum_{n=1}^{\infty} \frac{2 e^{-n \beta m}[1+2 \cos (n \theta)]}{n\left|1-e^{i n \theta}\right|^{2}} .
\end{aligned}
$$

Using now $\widehat{g}^{\mu \mu^{\prime}} \widehat{g}^{\nu \nu^{\prime}} \partial_{\mu} \partial_{\nu^{\prime}} \sigma \partial_{\nu} \partial_{\mu^{\prime}} \sigma=1+2 \cos (2 n \theta)$,

$$
\begin{aligned}
-\ln \operatorname{det}\left(\Delta^{(2)}-m^{2}\right) & =\int_{0}^{\infty} \frac{d t}{t} \sum_{n \in \mathbb{Z}} \int d^{3} x \sqrt{g} \widehat{g}^{\mu \mu^{\prime}} \widehat{g}^{\nu \nu^{\prime}} K_{\mu \nu, \mu^{\prime} \nu^{\prime}}\left(t, \sigma\left(x, \gamma^{n} x\right)\right) \\
& =\frac{5 m^{3}}{6 \pi} \operatorname{Vol}\left(\mathbb{R}^{3} / \mathbb{Z}\right)+\sum_{n=1}^{\infty} \frac{2 e^{-n \beta m}[1+2 \cos (n \theta)+2 \cos (2 n \theta)]}{n\left|1-e^{i n \theta}\right|^{2}} .
\end{aligned}
$$

Putting everything together according to (3.1), the one-loop correction to the classical Euclidean action is

$$
S^{(1)}=\sum_{n=1}^{\infty} \frac{2 e^{-n \beta m}[\cos (2 n \theta)-\cos (n \theta)]}{n\left|1-e^{i n \theta}\right|^{2}} \stackrel{m=0}{\longrightarrow} \sum_{n=1}^{\infty} \frac{1}{n}\left(\frac{e^{2 i n \theta}}{1-e^{i n \theta}}+\frac{e^{-2 i n \theta}}{1-e^{-i n \theta}}\right) .
$$

The two divergent sums can be made convergent by replacing $\theta$ by $\theta+i \epsilon$ in the first sum, and by $\theta-i \epsilon$ in the second one. ${ }^{1}$ Defining $q=e^{i(\theta+i \epsilon)}$ gives

$$
S^{(1)}=\sum_{n=1}^{\infty} \frac{1}{n}\left(\frac{q^{2 n}}{1-q^{n}}+\frac{\bar{q}^{2 n}}{1-\bar{q}^{n}}\right)
$$

or equivalently,

$$
e^{S^{(1)}}=\prod_{k=2}^{\infty} \frac{1}{\left|1-q^{k}\right|^{2}} .
$$

From (2.3) we can then read off the 1-loop partition function around flat space:

$$
Z[\beta, \theta]=e^{-\frac{1}{\hbar} S^{(0)}+S^{(1)}+O(\hbar)}=e^{\frac{\beta}{8 G \hbar}} \prod_{k=2}^{\infty} \frac{1}{\left|1-q^{k}\right|^{2}}(1+O(\hbar)) .
$$

This expression matches the vacuum $\mathrm{BMS}_{3}$ character, as computed in [8], for a Euclidean time translation by $\beta$ and central charge $c_{2}=3 / G$. It can also be obtained as the flat limit of the one-loop partition function in $\mathrm{AdS}_{3}[3]$.

\section{Acknowledgments}

We thank A. Lepage-Jutier, G. Moore, P. Salgado-Rebolledo and A. Strominger for useful conversations. This work is supported in part by the Fund for Scientific ResearchFNRS (Belgium), by IISN-Belgium, by "Communauté française de Belgique - Actions de Recherche Concertées" and by the National Science and Engineering Council of Canada.

\footnotetext{
${ }^{1}$ This analytic continuation is quite natural considering the fact that angular potentials which are real in Lorentzian signature become imaginary in Euclidean signature. The partition function $Z[\beta, \theta]$ is most naturally viewed as a function of complex angular potential.
} 
Open Access. This article is distributed under the terms of the Creative Commons Attribution License (CC-BY 4.0), which permits any use, distribution and reproduction in any medium, provided the original author(s) and source are credited.

\section{References}

[1] E. Witten, Three-dimensional gravity revisited, arXiv:0706.3359 [INSPIRE].

[2] A. Maloney and E. Witten, Quantum gravity partition functions in three dimensions, JHEP 02 (2010) 029 [arXiv:0712.0155] [INSPIRE].

[3] S. Giombi, A. Maloney and X. Yin, One-loop partition functions of 3D gravity, JHEP 08 (2008) 007 [arXiv:0804.1773] [INSPIRE].

[4] J.R. David, M.R. Gaberdiel and R. Gopakumar, The heat kernel on $A d S_{3}$ and its applications, JHEP 04 (2010) 125 [arXiv:0911.5085] [INSPIRE].

[5] J.D. Brown and M. Henneaux, Central charges in the canonical realization of asymptotic symmetries: an example from three-dimensional gravity, Commun. Math. Phys. 104 (1986) 207 [INSPIRE].

[6] A. Ashtekar, J. Bicak and B.G. Schmidt, Asymptotic structure of symmetry reduced general relativity, Phys. Rev. D 55 (1997) 669 [gr-qc/9608042] [INSPIRE].

[7] G. Barnich and G. Compere, Classical central extension for asymptotic symmetries at null infinity in three spacetime dimensions, Class. Quant. Grav. 24 (2007) F15 [gr-qc/0610130] [INSPIRE].

[8] B. Oblak, Characters of the BMS group in three dimensions, arXiv:1502.03108 [INSPIRE].

[9] G. Barnich and B. Oblak, Notes on the BMS group in three dimensions: I. Induced representations, JHEP 06 (2014) 129 [arXiv: 1403.5803] [INSPIRE].

[10] G. Barnich and B. Oblak, Notes on the BMS group in three dimensions: II. Coadjoint representation, JHEP 03 (2015) 033 [arXiv:1502.00010] [INSPIRE].

[11] S.M. Christensen and M.J. Duff, Quantizing gravity with a cosmological constant, Nucl. Phys. B 170 (1980) 480 [inSPIRE].

[12] T. Regge and C. Teitelboim, Role of surface integrals in the Hamiltonian formulation of general relativity, Annals Phys. 88 (1974) 286 [INSPIRE].

[13] M. Bañados, C. Teitelboim and J. Zanelli, The black hole in three-dimensional space-time, Phys. Rev. Lett. 69 (1992) 1849 [hep-th/9204099] [INSPIRE].

[14] G. Barnich, Entropy of three-dimensional asymptotically flat cosmological solutions, JHEP 10 (2012) 095 [arXiv:1208.4371] [INSPIRE].

[15] A. Bagchi, S. Detournay, R. Fareghbal and J. Simón, Holography of 3D flat cosmological horizons, Phys. Rev. Lett. 110 (2013) 141302 [arXiv: 1208.4372] [INSPIRE].

[16] G.T. Horowitz and A.R. Steif, Singular string solutions with nonsingular initial data, Phys. Lett. B 258 (1991) 91 [INSPIRE].

[17] A. Bagchi, S. Detournay, D. Grumiller and J. Simon, Cosmic evolution from phase transition of three-dimensional flat space, Phys. Rev. Lett. 111 (2013) 181301 [arXiv:1305.2919] [INSPIRE]. 
[18] S. Deser, R. Jackiw and G. 't Hooft, Three-dimensional Einstein gravity: dynamics of flat space, Annals Phys. 152 (1984) 220 [INSPIRE].

[19] K. Ezawa, Transition amplitude in $(2+1)$-dimensional Chern-Simons gravity on a torus, Int. J. Mod. Phys. A 9 (1994) 4727 [hep-th/9305170] [INSPIRE].

[20] L. Cornalba and M.S. Costa, A new cosmological scenario in string theory, Phys. Rev. D 66 (2002) 066001 [hep-th/0203031] [INSPIRE].

[21] L. Cornalba and M.S. Costa, Time dependent orbifolds and string cosmology, Fortsch. Phys. 52 (2004) 145 [hep-th/0310099] [INSPIRE].

[22] G. Barnich, A. Gomberoff and H.A. Gonzalez, The flat limit of three dimensional asymptotically anti-de Sitter spacetimes, Phys. Rev. D 86 (2012) 024020 [arXiv:1204.3288] [INSPIRE]. 